\title{
A PROOF OF THE MEAN ERGODIC THEOREM FOR NONEXPANSIVE MAPPINGS IN BANACH SPACE
}

\author{
NORIMICHI HIRANO
}

\begin{abstract}
Let $C$ be a closed convex subset of a uniformly convex Banach space. Let $T: C \rightarrow C$ be a nonexpansive mapping. In this paper, we deal with the weak convergence of the arithmetical means of the sequence $T^{n} x$, and give a new proof of the mean ergodic theorem for nonexpansive mappings.
\end{abstract}

1. Introduction. Let $X$ be a Banach space and $C$ be a closed convex subset of $X$. A mapping $T: C \rightarrow C$ is called nonexpansive on $C$ if

$$
\|T x-T y\| \leqslant\|x-y\| \text { for all } x, y \in C \text {. }
$$

Let $F(T)$ be the set of fixed points of $T$. If $X$ is strictly convex, $F(T)$ is closed and convex. In [1], Baillon proved the first nonlinear ergodic theorem such that if $X$ is a real Hilbert space and $F(T) \neq \Phi$, then for each $x \in C$, the sequence $\left\{S_{n} x\right\}$ defined by

$$
S_{n} x=(1 / n)\left(x+T x+\cdots+T^{n-1} x\right)
$$

converges weakly to a fixed point of $T$. It was also shown by Pazy [7] that if $X$ is a real Hilbert space and $S_{n} x$ converges weakly to $y \in C$, then $y \in F(T)$. These results were extended by Baillon [2], Bruck [4] and Reich [8], [9]. In this paper, we give a new proof of the following theorem which is due to Reich [9].

THEOREM. Let $X$ be a uniformly convex Banach space which has a Fréchet differentiable norm. Let $C$ be a closed convex subset of $X$ and $T: C \rightarrow C$ be $a$ nonexpansive mapping. Then the following conditions are equivalent:

(a) $F(T) \neq \Phi$

(b) $\left\{T^{n} x\right\}$ is bounded for each $x \in C$;

(c) for each $x \in C, S_{n} T^{i} x$ converges weakly to $y \in C$, uniformly in $i=1,2, \ldots$

2. Preliminaries. Let $X$ be a uniformly convex Banach space. The duality mapping $J$ of $X$ into $X^{*}$ is given by the conditions

$$
(J(x), x)=\|x\|^{2}, \quad\|J x\|=\|x\| .
$$

If $X$ is assumed to have a Fréchet differentiable norm, $J$ is continuous. co $D$ denotes the convex hull of $D, \overline{c o} D$ the closed convex hull of $D$. For $x, y \in X$, $[x, y]$ denotes the set $\{\lambda x+(1-\lambda) y: 0 \leqslant \lambda \leqslant 1\}$. Strong convergence is denoted by $\rightarrow$ and weak convergence is denoted by $\rightarrow$.

Received by the editors October 20, 1978 and, in revised form, February 14, 1979 and April 19, 1979. AMS (MOS) subject classifications (1970). Primary 47H10.

Key words and phrases. Nonexpansive mapping, fixed point theorem.

(c) 1980 American Mathematical Society 0002-9939/80/0000-0116/\$02.25 
The following lemmas are crucial for our discussion. The next lemma is known (cf. [6]). It is a simple consequence of the definition of the modulus of convexity.

LEMMA 1. Let $X$ be a uniformly convex Banach space with modulus of convexity $\delta$. Let $x, y \in X$. If $\|x\| \leqslant r,\|y\| \leqslant r, r \leqslant R$, and $\|x-y\| \geqslant \varepsilon>0$, then

$$
\|\lambda x+(1-\lambda) y\| \leqslant r\left(1-2 \lambda(1-\lambda) \delta_{R}(\varepsilon)\right)
$$

for all $\lambda: 0 \leqslant \lambda \leqslant 1$, where $\delta_{R}(\varepsilon)=\delta(\varepsilon / R)$.

Below, $X$ will denote a uniformly convex Banach space with modulus of convexity $\delta$.

LEMMA 2. Let $C$ be a closed convex subset of $X$ and $T: C \rightarrow C$ be a nonexpansive mapping. Let $x \in C, f \in F(T)$ and $0<\alpha \leqslant \beta<1$. Then for each $\varepsilon>0$, there exists $N>0$ such that for all $n \geqslant N$,

$$
\left\|T^{k}\left(\lambda T^{n} x+(1-\lambda) f\right)-\left(\lambda T^{n+k} x+(1-\lambda) f\right)\right\|<\varepsilon
$$

for all $k>0$ and $\lambda: \alpha \leqslant \lambda \leqslant \beta$.

Proof. Put $r=\lim _{n}\left\|T^{n} x-f\right\|, R=\|x-f\|$, and $c=\min \{2 \lambda(1-\lambda): \alpha<\lambda<$ $\beta$ \}. For given $\varepsilon>0$, choose $d>0$ so small that $r /(r+d)>1-c \delta_{R}(\varepsilon)$. Then there exists $N>0$ such that for all $n \geqslant N,\left\|T^{n} x-f\right\|\langle r+d$. For $n>N, k>0$ and $\alpha \leqslant \lambda \leqslant \beta$, we put $u=(1-\lambda)\left(T^{k} z-f\right)$ and $v=\lambda\left(T^{n+k} x-T^{k} z\right)$ where $z=\lambda T^{n} x+(1-\lambda) f$. Then $\|u\| \leqslant \lambda(1-\lambda)\left\|T^{n} x-f\right\|$ and $\|v\|<\lambda(1-\lambda) \| T^{n} x-$ $f \|$. Suppose that $\|u-v\|=\left\|T^{k} z-\left(\lambda T^{n+k} x+(1-\lambda) f\right)\right\| \geqslant \varepsilon$. Then by Lemma 1 ,

$$
\begin{aligned}
\|\lambda u+(1-\lambda) v\| & =\lambda(1-\lambda)\left\|T^{n+k} x-f\right\| \\
& \leqslant \lambda(1-\lambda)\left\|T^{n} x-f\right\|\left(1-2 \lambda(1-\lambda) \delta_{R}(\varepsilon)\right) \\
& \leqslant \lambda(1-\lambda)\left\|T^{n} x-f\right\|\left(1-c \delta_{R}(\varepsilon)\right)
\end{aligned}
$$

Hence we have $(r+d)\left(1-c \delta_{R}(\varepsilon)\right)<r \leqslant(r+d)\left(1-c \delta_{R}(\varepsilon)\right)$, which is a contradiction.

LEMMA 3 (BROWDER [3]). Let $C$ be a closed convex subset of $X$ and $T: C \rightarrow C$ be a nonexpansive mapping. If $\left\{u_{i}\right\}$ is a weakly convergent sequence in $C$ with weak limit $u_{0}$ and if $\lim _{i}\left\|u_{i}-T u_{i}\right\|=0$, then $u_{0}$ is a fixed point of $T$.

\section{Proof of Theorem.}

LEMMA 4. Let $C$ be a closed convex subset of $X$ and $T: C \rightarrow C$ be a nonexpansive mapping. Then for each $x \in C$ and each $n>0$,

$$
\lim _{i}\left\|T^{k} S_{n} T^{i} x-S_{n} T^{k} T^{i} x\right\|=0 \quad \text { uniformly in } k=1,2, \ldots
$$

Proof. The proof is by induction on $n$. First we prove in the case $n=2$. Put $r=\lim _{n}\left\|T^{n+1} x-T^{n} x\right\|, R=\|x-T x\|$ and $x_{i}=T^{i} x$ for $i=1,2, \ldots$ If $r \neq 0$, then for given $\varepsilon>0$, choose $c>0$ so small that $r /(r+c)>1-\delta_{R}(\varepsilon) / 2$. Then 
there exists $N>0$ such that for all $i \geqslant N,\left\|T^{k} x_{i}-T^{k+1} x_{i}\right\|<r+c$ for $k=$ $1,2, \ldots$ If we put $u=\frac{1}{2}\left(T^{k} z-T^{k} x_{i}\right)$ and $v=\frac{1}{2}\left(T^{k+1} x_{i}-T^{k} z\right)$ where $i>N$, $k>0$ and $z=\frac{1}{2}\left(x_{i}+T x_{i}\right)$, then

$$
\|u\| \leqslant \frac{1}{2}\left\|z-x_{i}\right\|=\frac{1}{4}\left\|T x_{i}-x_{i}\right\|<\frac{1}{4}(r+c) .
$$

Similarly, $\|v\| \leqslant \frac{1}{4}(r+c)$. Suppose that $\|u-v\|=\left\|T^{k} z-\frac{1}{2}\left(T^{k+1} x_{i}+T^{k} x_{i}\right)\right\|>$ $\varepsilon$. Then by Lemma 1,

$$
\left\|\frac{1}{2}(u+v)\right\|=\frac{1}{4}\left\|T^{k+1} x_{i}-T^{k} x_{i}\right\|<\frac{1}{4}(r+c)\left(1-\frac{1}{2} \delta_{R}(\varepsilon)\right),
$$

which contradicts $r>(r+c)\left(1-\frac{1}{2} \delta_{R}(\varepsilon)\right)$. If $r=0$, then for given $\varepsilon>0$, choose $i>0$ so large that $\|u\|<\varepsilon / 2$ and $\|v\|<\varepsilon / 2$. Hence we have $\| T^{k} z-\frac{1}{2}\left(T^{k+1} x_{i}+\right.$ $\left.T^{k} x_{i}\right)\|=\| u-v\|\leqslant\| u\|+\| v \|<\varepsilon$. This completes the proof of the case $n=2$. Now suppose that $\lim _{i}\left\|T^{k} S_{n-1} x_{i}-S_{n-1} T^{k} x_{i}\right\|=0$, uniformly in $k=1,2, \ldots$ We claim that $\lim _{i}\left\|S_{n-1} T x_{i}-x_{i}\right\|$ exists. Put $r=\lim \inf _{i}\left\|S_{n-1} T x_{i}-x_{i}\right\|$. Given $\varepsilon>0$, choose $i>0$ such that $\left\|S_{n-1} T x_{i}-x_{i}\right\|<r+\varepsilon / 2$ and $\| S_{n-1} T^{k} x_{i+1}-$ $T^{k} S_{n-1} x_{i+1} \|<\varepsilon / 2$. Then

$$
\begin{aligned}
\left\|S_{n-1} T x_{i+k}-x_{i+k}\right\| & \leqslant\left\|S_{n-1} T^{k} x_{i+1}-T^{k} S_{n-1} x_{i+1}\right\|+\left\|T^{k} S_{n-1} x_{i+1}-T^{k} x_{i}\right\| \\
& <\varepsilon / 2+r+\varepsilon / 2=r+\varepsilon, \text { for } k=1,2, \ldots
\end{aligned}
$$

Therefore

$$
\lim \sup _{i}\left\|S_{n-1} T x_{i}-x_{i}\right\|=\underset{k}{\lim \sup }\left\|S_{n-1} T x_{i+k}-x_{i+k}\right\|<r+\varepsilon .
$$

Since $\varepsilon$ is arbitrary, we have

$$
\lim \sup _{i}\left\|S_{n-1} T x_{i}-x_{i}\right\| \leqslant \lim _{i} \inf \left\|S_{n-1} T x_{i}-x_{i}\right\|,
$$

i.e., $\lim _{i}\left\|S_{n-1} T x_{i}-x_{i}\right\|$ exists. Now we put $r=\lim _{i}\left\|S_{n-1} T x_{i}-x_{i}\right\|$. If $r \neq 0$, for given $\varepsilon>0$, choose $c>0$ so small that $(r-c) /(r+2 c)>1-(2(n-$ $\left.1) / n^{2}\right) \delta_{3 r}(\varepsilon)$. Then there exists $N>0$ such that if $i>N,\left|\left\|S_{n-1} T x_{i}-x_{i}\right\|-r\right|<c$ and $\left\|S_{n-1} T^{k} x_{i+1}-T^{k} S_{n-1} x_{i+1}\right\| \leqslant c / n$. For $k>0$ and $i>N$, we put $u=(n /(n$ $-1))\left(T^{k} S_{n} x_{i}-T^{k} x_{i}\right)$ and $v=n\left(S_{n-1} T^{k} x_{i+1}-T^{k} S_{n} x_{i}\right)$. Then

$$
\begin{gathered}
\|u\| \leqslant\left\|S_{n-1} T x_{i}-x_{i}\right\| \leqslant r+c, \\
\|v\| \leqslant n\left\|S_{n-1} T^{k} x_{i+1}-T^{k} S_{n-1} x_{i+1}\right\|+\left\|S_{n-1} T x_{i}-x_{i}\right\|<r+2 c, \\
\|u-v\|=(n /(n-1))\left\|T^{k} S_{n} x_{i}-S_{n} T^{k} x_{i}\right\| .
\end{gathered}
$$

Hence by the method in the proof of the case $n=2$, we have $\left\|T^{k} S_{n} x_{i}-S_{n} T^{k} x_{i}\right\|$ $<\varepsilon$ for $k=1,2, \ldots$, and all $i \geqslant N$. If $r=0$, then as in the proof of the case $n=2$ there exists $N^{\prime}$ such that for each $i \geqslant N^{\prime},\|u\|<\varepsilon / 2$ and $\|v\|<\varepsilon / 2$. Therefore we have $\left\|T^{k} S_{n} x_{i}-S_{n} T^{k} x_{i}\right\|<\varepsilon$. This completes the proof.

We assume that the norm of $X$ is Fréchet differentiable. Then we have the following proposition.

Proposition 1 (CF. [4], [9]). Let $C$ be a closed convex subset of $X$ and $T: C \rightarrow C$ be a nonexpansive mapping. If we put $W(x)=\cap_{m} \overline{\operatorname{co}}\left\{T^{k} x: k \geqslant m\right\}$ for each $x \in C$, then $W(x) \cap F(T)$ is at most one point. 
Proof. Suppose that $f, g \in W(x) \cap F(T)$ and $f \neq g$. Put $h=(f+g) / 2$ and $r=\lim _{n}\left\|T^{n} x-g\right\|$. Since $h \in W(x),\|h-g\| \leqslant r$. For each $n$, we choose $p_{n} \in$ $\left[T^{n} x, h\right]$ such that

$$
\left\|p_{n}-g\right\|=\min \left\{\|y-g\|: y \in\left[T^{n} x, h\right]\right\} .
$$

By Theorem 2.5 of [5], $\left(J\left(g-p_{n}\right), p_{n}-T^{n} x\right) \geqslant 0$ where $J$ is the duality mapping. Since $p_{n} \in\left[T^{n} x, h\right]$, we have $\left(J\left(g-p_{n}\right), h-T^{n} x\right) \geqslant 0$. Suppose that

$$
\lim _{n} \inf \left\|p_{n}-g\right\|=\|h-g\| \text {. }
$$

Since $X$ is uniformly convex and $\left\|p_{n}-g\right\| \leqslant\left\|\left(p_{n}+h\right) / 2-g\right\|<\|h-g\|, p_{n}$ converges strongly to $h$. Since the duality mapping $J$ is norm-to-norm continuous, we have that for given $\varepsilon>0$, there exists $N>0$ such that $(J(g-h)-J(g-$ $\left.\left.p_{n}\right), h-T^{n} x\right) \geqslant-\varepsilon$, for all $n \geqslant N$. Therefore we have

$$
\begin{aligned}
\left(J(g-h), h-T^{n} x\right)= & \left(J(g-h)-J\left(g-p_{n}\right), h-T^{n} x\right) \\
& +\left(J\left(g-p_{n}\right), h-T^{n} x\right) \\
\geqslant & -\varepsilon+0=-\varepsilon .
\end{aligned}
$$

Then it follows that for each $y \in \cap_{m} \overline{\operatorname{co}}\left\{T^{k} x: k \geqslant m\right\},(J(g-h), h-y) \geqslant 0$. If we put $y=g$, we have $\|h-g\|=0$. This contradicts $h \neq g$. Suppose that $\lim \inf _{n}\left\|p_{n}-g\right\|<\|h-g\|$, then there exist $c>0$ and a subsequence $\left\{p_{n_{1}}\right\}$ of $\left\{p_{n}\right\}$ such that $\left\|p_{n_{i}}-g\right\|+c<\|h-g\|$. Put $p_{n_{i}}=\alpha_{i} T^{n_{i}} x+\left(1-\alpha_{i}\right) h$, for $i=$ $1,2, \ldots$ Then there exist $\alpha>0$ and $\beta<1$ such that $\alpha \leqslant \alpha_{i} \leqslant \beta$ for all $i$. By Lemma 2, there exists $N>0$ such that if $n \geqslant N$,

$$
\left\|T^{k}\left(\lambda T^{n} x+(1-\lambda) h\right)-\left(\lambda T^{n+k} x+(1-\lambda) h\right)\right\|<c
$$

for all $\lambda: \alpha \leqslant \lambda \leqslant \beta$ and for all $k>0$. If we choose $p_{n_{i_{0}}} \in\left\{p_{n_{i}}\right\}$ such that $n_{i_{0}} \geqslant N$, we have

$$
\begin{aligned}
\left\|p_{n_{i_{0}}+k}-g\right\| & =\left\|\left(\alpha_{i_{0}} T^{n_{i_{0}}+k} x+\left(1-\alpha_{i_{0}}\right) h\right)-g\right\| \\
& \leqslant\left\|T^{k} p_{n_{i_{0}}}-\left(\alpha_{i_{0}} T^{n_{i_{0}}+k} x+\left(1-\alpha_{i_{0}}\right) h\right)\right\|+\left\|T^{k} p_{n_{i_{0}}}-g\right\| \\
& <c+\left\|p_{n_{i_{0}}}-g\right\|<\|h-g\|
\end{aligned}
$$

for $k=1,2, \ldots$ Therefore we have $p_{n} \neq h$ for all $n \geqslant n_{i_{0}}$. It follows that $\left(J(g-h), h-T^{n} x\right) \leqslant 0$ for $n \geqslant n_{i_{0}}$. Then we have $(J(g-h), h-y) \leqslant 0$ for all $y \in \overline{\operatorname{co}}\left\{T^{k} x: k \geqslant n_{i_{0}}\right\}$. Put $y=f=h+(h-g)$, then $\|h-g\|=0$. This contradicts $h \neq g$.

Proof of Theorem. (a) $\Leftrightarrow$ (b) is known [3]. (c) $\Rightarrow(b)$ : Suppose that for some $x \in C$, there exists an unbounded subsequence $\left\{T^{n_{i}} x\right\}$ of $\left\{T^{n} x\right\}$. Since $T$ is nonexpansive, we have that for each $m>0$, the sequence $\left\{S_{m} T^{n_{1}} x\right\}$ is also unbounded. But this contradicts the condition (c). (b) $\Rightarrow(c)$ : Since $\left\{T^{n} x\right\}$ is bounded and

$$
\begin{aligned}
\left\|T S_{n} T^{i} x-S_{n} T^{i} x\right\| & \leqslant\left\|T S_{n} T^{i} x-S_{n} T T^{i} x\right\|+\left\|S_{n} T T^{i} x-S_{n} T^{i} x\right\| \\
& \leqslant\left\|T S_{n} T^{i} x-S_{n} T T^{i} x\right\|+(1 / n)\left\|T^{i+1+n} x-T^{i} x\right\|,
\end{aligned}
$$


there exists a sequence $\left\{S_{n} T^{i_{n}} x\right\}$ such that $\lim _{n}\left\|T S_{n} T^{i_{n} x}-S_{n} T^{i_{n}} x\right\|=0$. Then by Lemma 3 and Proposition 1, we have that any weakly convergent subsequence of $\left\{S_{n} T^{i_{n}} x\right\}$ converges weakly to $y$, i.e., $S_{n} T^{i_{n}} x \rightarrow y$ where $y=W(x) \cap F(T)$. Also by Lemma 4, we have that $\lim _{n}\left\|T S_{n} T^{i_{n}+k n+i} x-S_{n} T^{i_{n}+k n+i} x\right\|=0$, for all $i$ and $k$. Therefore we have that $S_{n} T^{i_{n}+k n} x_{i} \rightarrow y$ uniformly in $k=1,2, \ldots$. While for $n$ and $m: m \geqslant i_{n}$,

$$
S_{m} T^{i} x=\frac{1}{m} \sum_{k=0}^{m-1} T^{k} x_{i}=\frac{1}{m}\left(\sum_{k=i_{n}+j n}^{m-1} T^{k} x_{i}+n\left(\sum_{k=0}^{j} S_{n} T^{i_{n}+k n} x_{i}\right)+\sum_{k=0}^{i_{n}} T^{k} x_{i}\right)
$$

where $m=j n+i_{n}+r, r<n$. Since $\left\{S_{n} T^{i_{n}+k n} x_{i}\right\}$ converges to $y$ uniformly with respect to $k$, we have that $S_{m} T^{i} x$ converges weakly to $y$, uniformly in $i=1,2, \ldots$

ACKnowledgement. The author wishes to express his hearty thanks to Professor W. Takahashi for many suggestions and advice in the course of preparing the present paper.

\section{REFERENCES}

1. J. B. Baillon, Un théorème de type ergodique pour les contractions non linéaires dans un espace de Hilbert, C. R. Acad. Sci. Paris Sér. A-B 280 (1975), A1511-A1514.

2. __ Comportement asymptotique des itérés de contractions non linéaires dans les espaces $L_{p}, \mathbf{C} . \mathbf{R}$. Acad. Sci. Paris Sér. A-B 286 (1978), A157-A159.

3. F. E. Browder, Nonlinear operators and nonlinear equations of evolution in Banach spaces, Proc. Sympos. Pure Math., vol. 18, no. 2, Amer. Math. Soc., Providence, R. I., 1976.

4. R. E. Bruck, $A$ simple proof of the mean ergodic theorem for nonlinear contractions in Banach spaces, Israel J. Math. 32 (1979), 107-116.

5. F. R. Deutsch and P. H. Maserick, Applications of the Hahn-Banach theorem in approximation theory, SIAM Rev. 9 (1967), 516-530.

6. C. W. Groetsch, A note on segmenting Mann iterates, J. Math. Anal. Appl. 40 (1972), 369-372.

7. A. Pazy, On the asymptotic behavior of iterates of nonexpansive mappings in Hilbert space, Israel $\mathrm{J}$. Math. 26 (1977), 197-204.

8. S. Reich, Almost convergence and nonlinear ergodic theorems, J. Approximation Theory 24 (1978), 269-272.

9. Weak convergence theorems for nonexpansive mappings in Banach spaces, J. Math. Anal. Appl. 67 (1979), 274-276.

Department of Information Sciences, Tokyo Institute of Technology, OH-Okayama, MEGURO-KU, TOKYO, JAPAN 